\title{
Practices and Outcomes of Promoting the Competencies of Psychological Assistance Professionals in Defeating the COVID- 19 Pandemic
}

\author{
Xiaoming $\mathrm{Jia}^{1} \cdot$ Linyu $\mathrm{You}^{1} \cdot$ Qin $^{\mathrm{An}^{1}}$
}

Received: 21 August 2020/Accepted: 1 June 2021/Published online: 1 September 2021

(C) National Academy of Psychology (NAOP) India 2021

\begin{abstract}
The competencies of clinical or counseling psychologists influenced the effectiveness and qualities of the psychological assistance provided after the outbreak of the COVID-19 pandemic. The following practices were performed by the Clinical Psychology Registration Work Committee of the Chinese Psychological Society in promoting the competencies of the professionals: (1) Developed and introduced guidelines for psychological assistance provided via hotlines or the Internet. (2) Provided specific trainings based on the competency model constructed by knowledge, skills, and attitudes. These training programs included the psychological first aid under the major public health emergencies, crisis intervention, special methods and approaches for hotline and online services, related ethical guidelines, etc. (3) Emphasized on the supervision for the professionals worked front line. The professionals practiced effectively in dealing with suicide crisis, helping people who lost their loved one, and the adjustment to life during the quarantine. The practical experience showed that the working mechanism of issuing practical guidelines, providing specific training and supervision can effectively improve the competencies of the professionals and play an important role in the psychological assistance defeating the pandemic in China.
\end{abstract}

Qin An

anqin@bit.edu.cn

Xiaoming Jia

jiaxiaoming@bit.edu.cn

Linyu You

youlinyu0520@126.com

1 School of Humanities and Social Sciences, Beijing Institute of Technology, Beijing 100081, China
Keywords COVID-19 · Psychological assistance . Competency · Professional academic society ·

The clinical psychology registration work committee

\section{Introduction}

The COVID-19 pandemic is characterized by high infectivity, wide impact range, large range of people covered and high attention. According to the survey, this pandemic has caused widespread concern among the public, as well as fear, anger, sadness and panic (Wang, et al., 2020). Psychological assistance refers to the assistance provided by the psychological professionals to help the psychological reconstruction after major emergencies, which aimed at helping the affected people cope with the psychological concerns or trauma caused by the emergencies or disasters and gradually restoring the normal psychological status (Jia, 2009). According to the definition, psychological assistance was not limited to psychotherapy and counseling and involves multi-disciplinary works.

Normally, psychological hotlines recruited volunteers without strict requirements on the professional and academic background. However, the psychological assistance hotlines established during the pandemic were to provide professional services via hotlines or the Internet. It is actually a professional work of psychological counseling that has changed the way of working. In China, most of the counselors recruited by the psychological assistance hotlines at the time of the COVID-19 epidemic are certified and have related qualifications. They were more familiar with the face-to-face counseling and working through sessions, and were less familiar with single-session service via hotlines. Counselors competent for face-to-face counseling 
may not be able to cope with emergency services and crisis intervention under stress, or be qualified for hotline counseling and online text counseling, especially for real-life problems brought by the pandemic as well as special issues such as loss and grief. Psychological assistance during the epidemic required higher competencies of the counselors, and it is of great significance to improve the professional competence of psychological assistance professionals.

The Clinical Psychology Registration Work Committee of the Chinese Psychological Society is a self-regulated professional organization of psychological counseling and therapy appointed and appointed by the Executive Council of CPS to ensure the qualification of mental health service in 2007. The Work Committee released the Registration Criteria for Clinical and Counseling Psychology Organizations and Professionals the Chinese Psychological Society (1st edition); it was established in 2007 (the Chinese Psychological Society, 2007), and was revised in 2018 (the Chinese Psychological Society, 2018). It played an important role in promoting the professional development of China's psychological counseling and therapy. In face of the outbreak of the COVID-19 pandemic, the Work Committee was fully engaged in the psychological assistance. It assembled experts and developed the top-level design immediately. Combining with the specific demands of defeating the outbreak, the Work Committee provided various training, supervision, and issued a series of professional guidelines which anchored in enhancing the competencies of the psychological assistance professional. It made much explorations and practices creatively for how to provide psychological assistance normatively, orderly and effectively by a professional organization.

\section{Psychological Assistance Practices during the Pandemic}

Being competent means being able to carry out work according to professional standards (Barnett et al., 2006, p.61). As to psychological counseling, it requires the related knowledges, skills, abilities, and values needed to provide effective services (Barnett \& Johnson, 2008). Incompetent practices will greatly increase the possibility of causing harm to clients and significantly reduce the potential help for clients (Welfel, 2010). Due to the infectivity of the COVID-19, most of the psychological assistance were provided via hotlines or the Internet. As a result, the psychological assistance professionals need to learn how to adjust to the specific characteristics of the Internet or the hotlines, and they should be competent in areas of intervening the stress caused by the major public health emergencies. The Work Committee issued a series of emergency responses as guidelines for psychological assistance practices and ensured their competencies. Figure 1 illustrated the overall competency-enhance program designed by the Clinical Psychology Registration Work Committee.

\section{Issued Guidelines for Psychological Assistance, and Clarifying the Standards for Professional Practices during the Pandemic}

\section{Released Written Proposal for Defeating the COVID-19 as Professional Society Firstly}

The psychological intervention system under major public health emergencies is still developing. The Work Committee released written proposal for defeating the COVID19 as professional society at the very beginning on January 26th, based on the experience of previous emergency assistance, especially the experiences of the Sichuan earthquake, and also taking the seriousness and scope of the pandemic into consideration. The proposal calls for scientific, orderly, professionalized, and normative practices within the unified national framework for psychological assistance and coordinated by multiple departments. Besides, it reaffirmed adherence to professional ethics:

(1) Practice under the leadership and deployment of the National Health Commission, local governments and health commissions, and work in an orderly, efficient and stable manner;

(2) Take science as the criterion, professionals should actively participate in the psychological assistance arranged by the Work Committee or local, related organizations, related institutions orderly, including psychological hotline counseling, science popularization, editing disaster psychological intervention manual, and providing counseling/therapy or psychological assistance;

(3) Give full play to professional advantages, and provide professional supervision and training;

(4) Self-care is important as well. The professionals are encouraged to seek help from colleagues or supervisors when needed, and alternate work with rest.

The release of the proposal set the tone and provided guidelines for domestic psychological assistance, and had a positive impact nationally.

Issued Guidelines for Psychological Assistance Defeating the Pandemic

The hotlines and the Internet as the main approaches for psychological assistance, challenged the practitioners who were more familiar with face-to-face counseling. The professionals might lack related experiences or the 
Fig. 1 The overall competencyenhance program

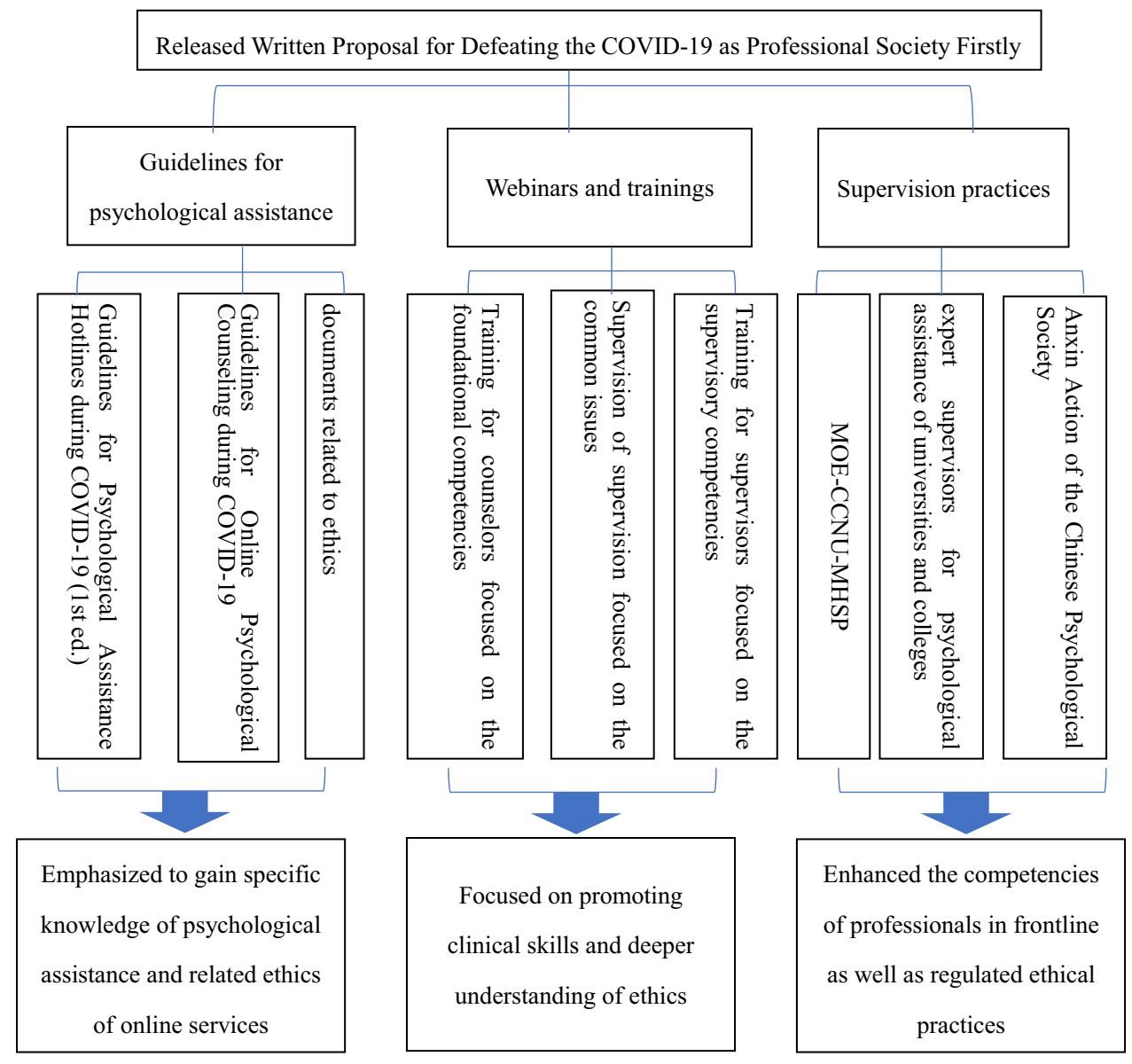

have formulated and promulgated the Ethical Codes for Hotline Counseling (1st ed., 2nd ed.), the Ethical Codes for Online Counseling, and the Eight Ethical Principles for Online Group Supervision, and so on. These ethical guidelines emphasized the competencies, informed consent, confidentiality and violations (e.g., the caller with diagnosis refused to get treatment), and the ethical demands for crisis intervention under the pandemic. These ethical documents provided instructions, norms, and guidelines for the psychological assistance professionals on the frontline national wide, and helped them to practice in a science-based, standardized way during the special period. The professional can practice ethically and competently when complying with these guidelines.

\section{Provided Formal Training based on the Competency Model}

\section{Training for Counselors Focused on the Foundational Competencies}

In order to promote the practical ability of frontline professionals, the Registration Work Committee organized two formal trainings for registered psychologists and ments related to ethics. The Registration Work Committee 
assistant psychologists based on the competency model, composed with knowledge, skills, and attitudes or values. These trainings focused on how to provide psychological assistance under the major public health emergencies, skills of crisis intervention, the specific techniques and approaches for online and hotline services, and related ethics (Table 1). Questions were raised by the professionals and fully discussed during the training, and over 1000 persontime attended. The attendees served in one or more major hotlines or platforms and the training covered almost all the provinces and autonomous regions in China. Besides, the experts of the Registration Work Committee provided thousands of trainings for different institutions or platforms over the country for psychological assistance during the pandemic, and played an important role in improving the professional competencies of psychological assistance in various fields.

\section{Supervision of Supervision Focused on the Common Issues}

There were few practitioners that participated in the psychological assistance during the SARS in 2003, and most of them lack the experience under major public health emergencies. As a result, it is strongly recommended to provide supervision to ensure the qualities and protect the clients' benefits. Besides, the supervisory competencies were required for the supervisors.

At the beginning of the outbreak, the Registration Work Committee, taking advantages of the expert resources, arranged a 7 day supervision for supervision with 14 experts. Each day, there were 2 experts on clinical supervision taking the host, and discussed specific topics including the development phases of psychological problems after disasters, crisis intervention via hotlines, and how to provide psychological assistance with the perspective of the Registration Work Committee, and so on (Table 2). The attendees can also raise questions from their own practical experience, and they can receive feedback and instructions instantly. There were person-time participated in the webinars, and they came from all over the country. All the supervisors in charge of supervision programs in 26 provinces and those who supervised in the psychological assistance hotlines/platforms attended. These webinars provided a space for full discussions, communications, and support for each other among the registered supervisors. With the directives of the experts, the psychological assistance work was carried out in a stable and orderly manner in different levels.

\section{Training for Supervisors Focused on the Supervisory Competencies}

To enhance the professional competence of registered supervisors and continue to play a leading role in the profession, the Registration Work Committee organized a five-day training course for registered supervisors. The supervision training team was led by five experts, and the training was focused on the supervision and supervisory theories, supervisory relationship and evaluation, individual supervision, group supervision, and ethics for supervision (Table 3). Besides, the experts answered on-site questions about the special difficulties of supervision during the pandemic. A total of 578 person-time participated in the training. All the supervisors in charge of supervision programs and those who supervised in the major psychological assistance hotlines/platforms attended.

As experts in psychological counseling and therapy, the registered supervisors were the backbone of psychological assistance. The competencies of the registered supervisors were improved, and the confidence, sense of responsibility, and cohesion among them were enhanced at the same time. They provided supervisions, trainings and consultations on crisis intervention and hotline counseling for the professional assistance professionals at the front line across the country, and played an important role in the psychological assistance in fighting the pandemic. With the pandemic reaching a stationary phase in China, the supervisors' competencies have been further improved and they are ready to provide regular assistance continuously during the post-pandemic period.

\section{Enhance the Supervision for the Professionals on the Front Line}

\section{Pyramid Model for Supervision}

As to the implemented level of psychological assistance, the Registration Work Committee creatively designed the pyramid model for supervision based on the expert resources (Fig. 2). The first level referred to professionals

Table 1 Competence-based training for Registered Psychologists of CPS

\begin{tabular}{ll}
\hline Date & Theme \\
\hline Feb. 7th & Interpretation of Working Guidelines for Psychological Assistance Hotlines \\
Feb. 9th & Basic Skills and Relationship Building in psychological Assistance Hotlines \\
\hline
\end{tabular}


Table 2 Online Consultations for Registered Supervisors of CPS

\begin{tabular}{llc}
\hline Date & Main Topic & Number of Attendees \\
\hline Jan. 28th & The Rational Attitudes and Multi-Cultural Sensitivities in Psychological Assistance & 120 \\
Jan. 29th & The Functions and Responsibilities of Supervision for Crisis Intervention & 105 \\
Jan. 30th & Intervention Based on the Different Categories and Stages and Related Ethical Issues & 126 \\
Jan. 31th & Differentiating the Stress Response from the Pre-exiting Mental Disorders & 124 \\
Feb. 1st & Supervision for Marriage and Family Counseling during the Pandemic & 130 \\
Feb. 2nd & Competencies of the Supervisees and Ethical Issues Related to the Hotlines & 128 \\
Feb. 3rd & How to Deal with Sexual Harassing Calls and Supervision Strategies for Hotlines & 126 \\
\hline
\end{tabular}

Table 3 Training for Registered Supervisors of CPS

\begin{tabular}{lll}
\hline Date & Theme & Number of Attendees \\
\hline Feb. 10th & Introduction to Supervision Models & 113 \\
Feb. 11th & Evaluation in Supervision and Supervisory Relationships & 110 \\
Feb. 12th & Individual Supervision & 114 \\
Feb. 13th & Group Supervision & 116 \\
Feb. 14th & Supervisory Ethics & 125 \\
\hline
\end{tabular}

Fig. 2 The pyramid supervision model

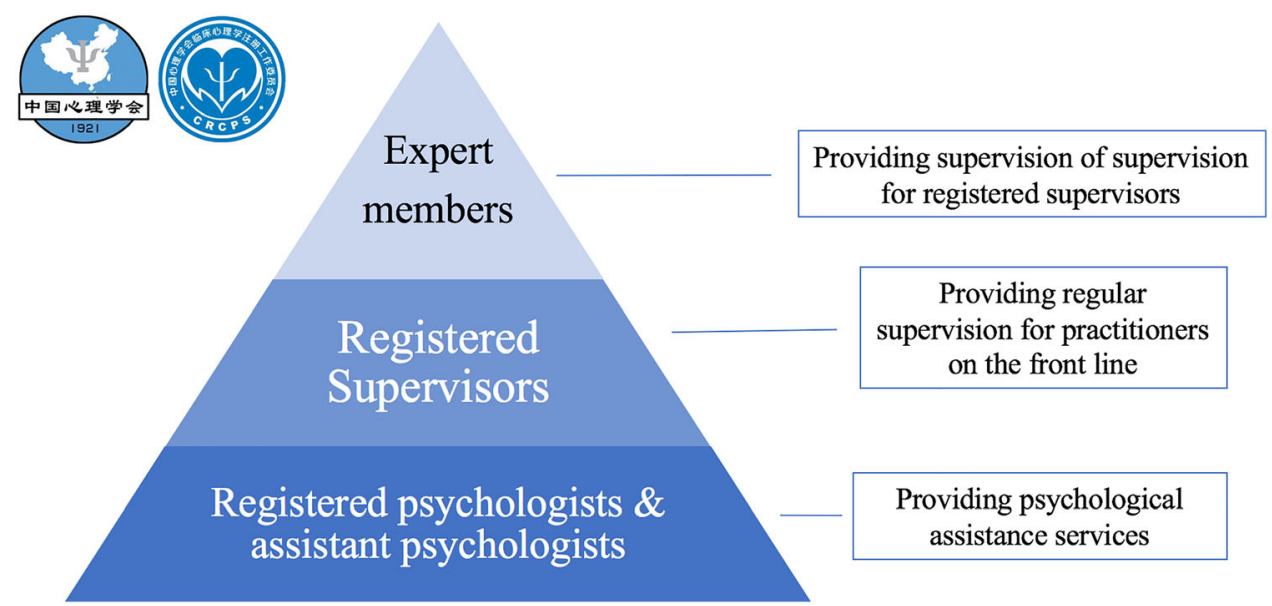

who provide psychological assistance at the front line; the second level meant the registered supervisors providing supervision for the psychological assistance professionals; on the top level, the Registration Work Committee organized expert members to provide supervision's supervision for registered supervisors. This model ensured the psychological assistance carried out in a hierarchical and orderly manner.

\section{Main Supervision Practices}

One of the major works of the Registration Work Committee was providing clinical supervisions for the psychological assistance professionals. On January 30, the
Registration Work Committee published a list of more than 150 registered supervisors who were willing to provide supervisions for free on the official Wechat account, which made them available for practitioners in need. Nearly 200 supervisors provided clinical supervision for the institutions or platforms national wide in individual and group settings, ensured the effectiveness and qualities of the services provided, and safeguarded the interests of those seeking help.

Supporting the Mental Health Service Platform at Central China Normal University, Ministry of Education (MOE-CCNU-MHSP) The MOE-CCNU-MHSP is funded by Ministry of Education (MOE) and based on the School of Psychology at Central China Normal University 
(CCNU). The Registration Work Committee work, as one of the core support unit, provided the maximum resources. The Committee arranged a supervision group consisted with 12 expert members to provide supervision for supervision, and all of the 124 supervisors working with the counselors were registered supervisors. Besides, there were more than 1700 counselors that provided online counseling and hotline counseling, and most of them were registered psychologists and assistant psychologists. The Work Committee introduced the three level of professionalsSupervisor's supervisor + Supervisor + Psychologists-into the platform and effectively ensured the qualities of the hotline service.

Organized expert supervisors for psychological assistance of universities and colleges The Registration Work Committee collaborated with the Professional Committee for College Students Psychological Counseling of the Chinese Association for Mental Health and organized the expert members from the Registration Work Committee. There were six supervisor groups who provide supervision according to the needs of different universities to carry out high-quality clinical supervision in the six regions of the country.

General supervision for the "Fight against the Epidemic and Help to Release Action (Anxin Action)" of the Chinese Psychological Society As the member of the supervision expert team, the experts from the Registration Work Committee provided supervision and supervision for supervision for the volunteers recruited. The volunteers had varied training backgrounds and qualifications, and also varied in the competency level. As a result, there were much corrective interventions for the counselors' nonprofessional behaviors.

\section{Effects and Outcomes: Take the MOE-CCNU- MHSP for Example}

The MOE-CCNU-MHSP was funded on January 31st, providing psychological assistance based on the Internet platform in form of hotlines or text. According to their report (Wu \& Jiang, 2020), by July 29th, more than 18,551 person-times counselors had been assigned and 53,512 person-times had been called in and 31,271 person-times had been answered. There were 547 crisis intervention cases and 609 overseas students/overseas Chinese seek for help. The 124 registered supervisors of the Registration Work Committee are fully responsible for the supervision and training of the psychological assistance of the MOECCNU-MHSP. The platform adopts a three-level supervision structure. The first level contained counselors providing psychological assistance directly to the callers on duty. The second level were registration supervisors providing regular supervision to the counselors, and the expert supervisors formed the third level providing supervision of supervision. Besides, there were quality control and process regulations to ensure the effective operation of supervision.

In the past six months, the platform has implemented a total of 1087 supervision sessions including individual supervision, group supervision, supervision for supervision and case conferences with a total length of $1318 \mathrm{~h}$, covering 23,599 person-times. According to the feedback from counselors, the focus of supervision was on psychological assistance skills (22.44\%), self-care issues (16.51\%), crisis intervention issues $(15.12 \%)$, psychological assistance ethics $(13.87 \%)$ and knowledge on psychological assistance $(13.81 \%)$, etc. $22.55 \%$ of the counselors impressed mostly by the emotional support/empowerment of supervisors, followed by skill guidance/norms (17.10\%) and assistance/discussion on improving self-awareness (16.43\%). The average score of supervisors rated by the counselors is over 8.9 (out of 10). In addition, based on a satisfaction survey of 2543 callers, the vast majority of callers felt understood, helped and satisfied with the conversation sessions, with 93.2 percent of callers' satisfaction (Wu \& Jiang, 2020).

The platform was co-led by the Clinical and Counseling Psychology Committee of the Chinese Psychological Society together with the Registration Work Committee, and the expert team promoted and participated in the design and operational management of supervision. The pyramid supervision model functioned well and the quality of supervision was ensured. The feedback was collected continuously to adjust and improve the professional services, which improved the counselors' competencies, and at the same time, the supervisors were instructed by the experts on how to empower the counselors. The professionals successfully dealt with crisis interventions, helped the grief of the bereaved person, and how to adapt to the quarantine period. The quality of psychological assistance service was guaranteed to the greatest extent (Wu \& Jiang, 2020).

\section{Conclusions and Reflections}

Hotlines and online services become the major approaches for psychological assistance under the major infectious public health emergencies, thus, specific competencies were required to provide qualified services. After the outbreak of COVID-19, the most important work done by the Registration Work Committee is to establish standards leading the profession to conduct psychological assistance in a scientific, orderly and standardized way. To fulfill its mission and responsibilities, the Registration Work 
Committee explored the psychological intervention mechanism for major public health emergencies under the current professionalized phase of psychological counseling in China.

At the very beginning of the pandemic, the Registration Work Committee issued practical guidelines and ethical norms based on a combination of competency model and the characteristics of the pandemic, to help overcome the confusion and difficulties faced by the professionals. In addition, facilitating the expert members to provide supervision, supervision of supervision and consultations for the psychological assistance professionals working in the hotlines or online platforms. These practices ensured the qualities of psychological services, avoid harmless, and further help the clients. Besides, the Registration Work Committee gathered the top experts from the profession and held a series of trainings including ethics and implemented effective management mode. These practices actively promoted and supported psychological assistance across the country, emphasized the ethics for psychological assistance, and explored an effective way to provide psychological assistance applied to the Chinese culture and the existing realities in China.

The Registration Work Committee put the registered supervisors in a central position and provided essential training and support for them to help supervise the psychological assistance counselors. It formed the Butterfly Effect and guaranteed their competencies effectively. In addition, the competencies of professionals at different levels have also been improved in the practice of psychological assistance against the pandemic. The crisis caused by the pandemic has turned into an opportunity for professional development, forming a positive feedback cycle.

The psychological assistance will continue, and sustained psychological assistance is needed for the recovery and reconstruction after the pandemic. The Registration Work Committee further reflected on and looked forward to psychological assistance:

(1) The Registration Work Committee shall further optimize the professional norms for psychological assistance in the case of major disasters or emergencies, and develop detailed implementation rules for different types of psychological assistance, such as natural disasters, public health emergencies and man-made disasters, including the content, procedures and methods of specific psychological assistance. At the same time, the corresponding ethical norms should be established.

(2) Make use of professional resources to strengthen the competency training of the professionals for psychological assistance in emergencies, including knowledge, skills and ethics, and reserve a professional psychological assistance workforce composed by supervisors and practitioners for future disasters.

(3) As a professional academic organization, the Registration Work Committee actively cooperate with the government during the pandemic by offering suggestions and advice from the professional perspective. Such practices will greatly promote the standardization and specialization of psychological assistance.

In conclusion, the practical experience in organizing psychological assistance by the Clinical Psychology Registration Work Committee of the Chinese Psychological Society illustrated how one academic organization with national influences could do to direct and regulate the overall psychological assistance. Besides, the cooperation between academic organizations and national government was quite essential and effective, especially when considering the national governance system and the immature professionalism of psychological counseling and therapy in China.

Authors' Contributions All authors contributed to the study conception and design. JXM and AQ mainly designed the structure of the manuscript, provided related materials and critically revised the manuscript collaboratively. YLY performed the literature search and the translation work.

Funding Not Applicable.

\section{Declarations}

Conflicts of interest The authors declare that they have no have conflict of interest.

Ethics Approval Ethical review and approval was not required for this study because this is a review and no participants were involved.

\section{References}

Barnett, J. E., Johnston, L. C., \& Hillard, D. (2006). Psychological wellness as an ethical imperative. In L. Vande Creek \& J. B. Allen (Eds.), Innovations in clinical practice: Focus on health and wellness. Professional Resources Press.

Barnett, J. E., \& Johnson, W. B. (2008). Ethics desk reference for psychologists. American Psychological Association.

Chinese Psychological Society. (2007). The registration criteria for clinical and counseling psychology organizations and professionals of the Chinese Psychological Society (1st edition). Acta Psychologica Sinica, 39(5), 942-946.

Chinese Psychological Society. (2018). The registration criteria for clinical and counseling psychology organizations and professionals of the Chinese Psychological Society (2nd edition). Acta Psychologica Sinica, 50(11), 1303-1313.

Jia, X. M. (2009). New perspectives of psychological assistance after earthquake. China Journal of Health Psychology, 7, 882-885.

Wu, C. Z., \& Jiang, G. R. (2020). A three-layer supervision model of MOE-CCNU mental health service platform. Symposium 
conducted at the meeting of the 14th International Interdisciplinary Conference on Clinical Supervision.

Welfel, E. R. (2010). Ethics in Counseling and Psychotherapy Chinese version translated by Hou ZJ et al. (3rd ed.). World Publishing Corporation.

Wang, J. X., Gao, W. J., Chen, M. Q., Ying, X. P., Tan, X. Y., \& Liu, X. L. (2020). A survey of social attitudes in the context of
COVID-19. https://mp.weixin.qq.com/s/KH7Hfx-NnJQcJwWw_ jc_IA

Publisher's Note Springer Nature remains neutral with regard to jurisdictional claims in published maps and institutional affiliations. 